\title{
EXISTENCE RESULTS FOR FOURTH-ORDER ELASTIC BEAM EQUATIONS ON THE REAL LINE
}

\author{
MOHAMMAD REZA HEIDARI TAVANI \\ Department of Mathematics \\ Ramhormoz Branch \\ Islamic Azad University \\ Ramhormoz, IRAN
}

\begin{abstract}
The existence and multiplicity of solutions for a perturbed fourthorder problem on the real line with a perturbed nonlinear term depending on one real parameter is investigated. Our approach is based on variational methods and critical point theory which are obtained in [3].
\end{abstract}

AMS Subject Classification: 34B40, 34B15, 47H14

Key Words: multiplicity results, non-trivial solution, critical point theory, variational methods

Received: 2017-09-01;

Published: January 26, 2018
Accepted: 2017-12-15;

doi: $\quad 10.12732 /$ dsa.v27i1.8

\section{INTRODUCTION}

In this paper we consider the following problem

$$
u^{i v}(x)+A u^{\prime \prime}(x)+B u(x)=\lambda \alpha(x) f(u(x)), \text { a.e. } x \in \mathbb{R},
$$

where $A$ is a real negative constant and $B$ is a real positive constant, $\lambda$ is a positive parameter and $\alpha, f: \mathbb{R} \rightarrow \mathbb{R}$ are two functions such that $\alpha \in L^{1}(\mathbb{R}), \alpha(x) \geq 0$, for a.e. $x \in \mathbb{R}, \alpha \neq \equiv 0$ and also $f$ is continuous and non-negative.

It is well known that fourth-order problems are important in describing a large class of elastic deflections. Hence, many researchers have studied the existence and multiplicity of solutions for fourth-order two-point boundary value problems. We refer the reader to $[5,6,7,9,10,12]$. 
In [5], while $A$ and $B$ are real constants, using variational methods and critical point theory, multiplicity results for the fourth-order elliptic problem

$$
\left\{\begin{array}{l}
u^{i v}+A u^{\prime \prime}+B u=\lambda f(t, u), \\
u(0)=u(1)=u^{\prime \prime}(0)=u^{\prime \prime}(1)=0
\end{array} \quad t \in[0,1]\right.
$$

by condition on the nonlinear term was established, while in [9], applying the Morse theory, the existence of three solutions to problem (1), with $A=B=0$, were discussed.

Problems such as $\left(P_{\lambda}\right)$ that are discussed on the whole space, occur naturally in a variety of settings in physics and material scinces, as in, for example, the study of mathematical models of deflection of beams. These beams which appear in many structures, deflect under their own weight or under the influence of some external forces. Due to the lack of compactness of the operators on whole space, the study of such problems is very important. Because, in such cases the operators which solve the problem are not regular enough in comparison to operators which arise in problems on bounded domains.

In the present paper, using two kinds of critical point theorems obtained in [3] which we recall in the next section (Theorems 2.3 and 2.4), we establish the existence of at least one non-trivial and non-negative weak solution for the problem $\left(P_{\lambda}\right)$.

We must note that the importance of using the Theorem 2.3 in relation to the Theorem 2.5 in [11] is that, first, the sequential weak lower semi-continuity of $I_{\lambda}$ (the functional related to the problem $\left(P_{\lambda}\right)$ ) is not required and, secondly, the local minimum is non-trivial. Also, in presenting Theorem 3.3, which one of the main results of this paper, we aplly the requirements (Palais-Smale condition and unbounded from below for functional $I_{\lambda}$ ) based on Theorem 2.4. In fact, using one of the types of Ambrosetti-Rabinowitz conditions obtained in [1], we will ensure that functional $I_{\lambda}$ is unbounded from below.

\section{PRELIMINARIES}

Let us recall some basic consepts.

Denote $W_{0}^{2,2}(\mathbb{R})$ is the closure of $C_{0}^{\infty}(\mathbb{R})$ in $W^{2,2}(\mathbb{R})$ and it is clearly that $W_{0}^{2,2}(\mathbb{R})=$ $W^{2,2}(\mathbb{R})$. We denote by $|\cdot|_{t}$ the usual norm on $L^{t}(\mathbb{R})$, for all $t \in[1,+\infty]$ and it is well known that $W^{2,2}(\mathbb{R})$ is continuously embedded in $L^{t}(\mathbb{R})$ for each $t \in[2,+\infty]$.

The sobolev space $W^{2,2}(\mathbb{R})$ is equipped with the following norm

$$
\|u\|_{W^{2,2}(\mathbb{R})}=\left(\int_{\mathbb{R}}\left(\left|u^{\prime \prime}(x)\right|^{2}+\left|u^{\prime}(x)\right|^{2}+|u(x)|^{2}\right) d x\right)^{1 / 2},
$$


for all $u \in W^{2,2}(\mathbb{R})$.Also, we consider $W^{2,2}(\mathbb{R})$ with the norm

$$
\|u\|=\left(\int_{\mathbb{R}}\left(\left|u^{\prime \prime}(x)\right|^{2}-A\left|u^{\prime}(x)\right|^{2}+B|u(x)|^{2}\right) d x\right)^{1 / 2},
$$

for all $u \in W^{2,2}(\mathbb{R})$. According to

$$
(\min \{1,-A, B\})^{\frac{1}{2}}\|u\|_{W^{2,2}(\mathbb{R})} \leq\|u\| \leq(\max \{1,-A, B\})^{\frac{1}{2}}\|u\|_{W^{2,2}(\mathbb{R})},
$$

the norm $\|$. $\|$ is equvalent to the norm $\|.\|_{W^{2,2}(\mathbb{R})}$. Since embedding $W^{2,2}(\mathbb{R}) \rightarrow$ $L^{\infty}(\mathbb{R})$ is continuous hence there exists a constant $C_{A, B}$ (depending on A and B) such that

$$
|u|_{\infty} \leq C_{A, B}\|u\| \quad, \forall u \in W^{2,2}(\mathbb{R})
$$

In the following proposition, we provide an approximation for this constant.

Proposition 2.1. We have,

$$
|u|_{\infty} \leq C_{A, B}\|u\|
$$

where $C_{A, B}=\left(\frac{-1}{4 A B}\right)^{\frac{1}{4}}$.

Proof. let $v \in W^{1,1}(\mathbb{R})$, then from [[8]-P.138-formula 4.64],one has

$$
|v(x)| \leq \frac{1}{2} \int_{\mathbb{R}}\left|v^{\prime}(t)\right| d t .
$$

Now if $u \in W^{2,2}(\mathbb{R})$ then $v(x)=(-A B)^{\frac{1}{2}}|u(x)|^{2} \in W^{1,1}(\mathbb{R})$ and thus from (3) and Hölder's inequality one has,

$$
(-A B)^{\frac{1}{2}}|u(x)|^{2} \leq \int_{\mathbb{R}}(-A B)^{\frac{1}{2}}\left|u^{\prime}(t)\right||u(t)| d t \leq\left((-A)^{\frac{1}{2}}\left|u^{\prime}\right|_{2}\right)\left(B^{\frac{1}{2}}|u|_{2}\right)
$$

that is,

$$
|u(x)| \leq\left(\frac{-1}{A B}\right)^{\frac{1}{4}}\left((-A)^{\frac{1}{2}}\left|u^{\prime}\right|_{2}\right)^{\frac{1}{2}}\left(B^{\frac{1}{2}}|u|_{2}\right)^{\frac{1}{2}} .
$$

Now according to $x^{a} y^{1-a} \leq a^{a}(1-a)^{1-a}(x+y), x, y \geq 0,0<a<1$ ([[8],p.130,formula 4.47]), and classical inequality $a^{\frac{1}{p}}+b^{\frac{1}{p}} \leq 2^{\frac{(p-1)}{p}}(a+b)^{\frac{1}{p}}$, from (4) one has

$$
\begin{gathered}
|u(x)| \leq\left(\frac{-1}{A B}\right)^{\frac{1}{4}}\left(\frac{1}{2}\right)^{\frac{1}{2}}\left(\frac{1}{2}\right)^{\frac{1}{2}}\left[\left(\int_{\mathbb{R}}-A\left|u^{\prime}(t)\right|^{2} d t\right)^{\frac{1}{2}}+\left(\int_{\mathbb{R}} B|u(t)|^{2} d t\right)^{\frac{1}{2}}\right] \leq \\
\left(\frac{-1}{A B}\right)^{\frac{1}{4}}\left(\frac{1}{2}\right)^{\frac{1}{2}}\left(\frac{1}{2}\right)^{\frac{1}{2}}(2)^{\frac{1}{2}}\left(\int_{\mathbb{R}}\left(-A\left|u^{\prime}(t)\right|^{2}+B|u(t)|^{2}\right) d t\right)^{\frac{1}{2}} \leq \\
\left(\frac{-1}{4 A B}\right)^{\frac{1}{4}}\left(\int_{\mathbb{R}}\left(\left|u^{\prime \prime}(t)\right|^{2}-A\left|u^{\prime}(t)\right|^{2}+B|u(t)|^{2}\right) d t\right)^{\frac{1}{2}}
\end{gathered}
$$

which means that, $|u|_{\infty} \leq C_{A, B}\|u\|$. 
Let $\Phi, \Psi: W^{2,2}(\mathbb{R}) \rightarrow \mathbb{R}$ be defined by

$$
\Phi(u)=\frac{1}{2}\|u\|^{2}=\frac{1}{2} \int_{\mathbb{R}}\left(\left|u^{\prime \prime}(x)\right|^{2}-A\left|u^{\prime}(x)\right|^{2}+B|u(x)|^{2}\right) d x
$$

and

$$
\Psi(u)=\int_{\mathbb{R}} \alpha(x) F(u(x)) d x
$$

for every $u \in W^{2,2}(\mathbb{R})$ where $F(t)=\int_{0}^{t} f(\xi) d \xi$ for all $t \in \mathbb{R}$. Since $F^{\prime}(t)=f(t) \geq 0$ for all $t \in \mathbb{R}$ so $F$ is an increasing function. It is clear that $\Psi$ is well defined. Because for every $u \in W^{2,2}(\mathbb{R})$ we have

$$
|\Psi(u)| \leq \int_{\mathbb{R}} \alpha(x) \cdot \max \left\{-F\left(-|u|_{\infty}\right), F\left(|u|_{\infty}\right)\right\} d x<+\infty .
$$

It is well known that $\Psi$ is a differentiable functional whose differential at the point $u \in W^{2,2}(\mathbb{R})$ is

$$
\Psi^{\prime}(u)(v)=\int_{\mathbb{R}} \alpha(x) f(u(x)) v(x) d x,
$$

and, $\Phi$ is continuously Gâteaux differentiable functional whose differential at the point $u \in W^{2,2}(\mathbb{R})$ is

$$
\Phi^{\prime}(u)(v)=\int_{\mathbb{R}}\left(u^{\prime \prime}(x) v^{\prime \prime}(x)-A u^{\prime}(x) v^{\prime}(x)+B u(x) v(x)\right) d x
$$

for every $v \in W^{2,2}(\mathbb{R})$.

Definition 2.1. Let $\Phi$ and $\Psi$ be defined as above. Put $I_{\lambda}=\Phi-\lambda \Psi, \lambda>0$. We say that $u \in W^{2,2}(\mathbb{R})$ is a critical point of $I_{\lambda}$ when $I_{\lambda}^{\prime}(u)=0_{\left\{W^{2,2}(\mathbb{R})^{*}\right\}}$, that is, $I_{\lambda}^{\prime}(u)(v)=0$ for all $v \in W^{2,2}(\mathbb{R})$.

Definition 2.2. A function $u: \mathbb{R} \rightarrow \mathbb{R}$ is a weak solution to the problem $\left(P_{\lambda}\right)$ if $u \in W^{2,2}(\mathbb{R})$ and

$$
\int_{\mathbb{R}}\left(u^{\prime \prime}(x) v^{\prime \prime}(x)-A u^{\prime}(x) v^{\prime}(x)+B u(x) v(x)-\lambda \alpha(x) f(u(x)) v(x)\right) d x=0,
$$

for all $v \in W^{2,2}(\mathbb{R})$.

Remark 2.1. We clearly observe that the weak solutions of the problem $\left(P_{\lambda}\right)$ are exactly the solutions of the equation $I_{\lambda}^{\prime}(u)(v)=\Phi^{\prime}(u)(v)-\lambda \Psi^{\prime}(u)(v)=0$. Also if $\alpha$ is, in addition, a continuous function on $\mathbb{R}$ then each weak solution of $\left(P_{\lambda}\right)$ is a classical solution.

Lemma 2.2. If $u_{0} \not \equiv 0$ is a weak solution for problem $\left(P_{\lambda}\right)$ then $u_{0}$ is non-negative. 
Proof. From remark 2.1 one has, $I_{\lambda}^{\prime}\left(u_{0}\right)(v)=0$ for all $v \in W^{2,2}(\mathbb{R})$. Chossing $v(x)=\bar{u}_{0}=\max \left\{-u_{0}(x), 0\right\}$ and let $A=\left\{x \in \mathbb{R}: u_{0}(x)<0\right\}$. Then we have

$$
\int_{A \cup A^{c}}\left(u_{0}^{\prime \prime}(x) \bar{u}_{0}^{\prime \prime}(x)-A u_{0}^{\prime}(x) \bar{u}_{0}^{\prime}(x)+B u_{0}(x) \bar{u}_{0}(x)\right) d x=\int_{A \cup A^{c}} \lambda \alpha(x) f(u(x)) v(x) d x
$$

that is

$$
-\int_{A}\left(\left|\bar{u}_{0}^{\prime \prime}(x)\right|^{2}-A\left|\bar{u}_{0}^{\prime}(x)\right|^{2}+B\left|\bar{u}_{0}(x)\right|^{2}\right) d x=\int_{A} \lambda \alpha(x) f(u(x)) v(x) d x \geq 0
$$

which means that $-\left\|\bar{u}_{0}\right\|^{2} \geq 0$ and one has, $\bar{u}_{0}=0$. Hence $-u_{0} \leq 0$, that is, $u_{0} \geq 0$ and the proof is complete.

Definition 2.3. (see [2]) Fix $r \in]-\infty,+\infty]$. A Gâtuax differentiable function $I$ satisfies the Palais-Smale condition cut off upper at $r$ (in short $(P S)^{[r]}$-condition) if any sequence $\left\{u_{n}\right\}$ such that:

(a) $\left\{I\left(u_{n}\right)\right\}$ is bounded,

(b) $\lim _{n \rightarrow+\infty}\left\|I^{\prime}\left(u_{n}\right)\right\|_{X^{*}}=0$,

(c) $\Phi\left(u_{n}\right)<r \quad \forall n \in \mathbb{N}$,

has a convergent subsequence.

Definition 2.4. A Gâtuax differentiable function $I$ satisfies the Palais-Smale condition (in short $(P S)$-condition) if any sequence $\left\{u_{n}\right\}$ such that:

(a) $\left\{I\left(u_{n}\right)\right\}$ is bounded,

(b) $\lim _{n \rightarrow+\infty}\left\|I^{\prime}\left(u_{n}\right)\right\|_{X^{*}}=0, \quad \forall n \in \mathbb{N}$,

has a convergent subsequence.

Our main tools are the following critical point theorems.

Theorem 2.3 ([3], Theorem 2.3). Let $X$ be a real Banach space, and let $\Phi, \Psi$ : $X \longrightarrow \mathbb{R}$ be two continuously Gâteaux differentiable functionals such that $\inf _{X} \Phi=$ $\Phi(0)=\Psi(0)=0$.

Assume that there are $r \in \mathbb{R}$ and $\tilde{u} \in X$, with $0<\Phi(\tilde{u})<r$, such that

$$
\frac{\sup _{u \in \Phi^{-1}(]-\infty, r[)} \Psi(u)}{r}<\frac{\Psi(\tilde{u})}{\Phi(\tilde{u})},
$$

and, for each $\lambda \in] \frac{\Phi(\tilde{u})}{\Psi(\tilde{u})}, \frac{r}{\sup _{u \in \Phi^{-1}(]-\infty, r[)} \Psi(u)}[$ the functional $\Phi-\lambda \Psi$ satisfies the $(P S)^{[r]}$-condition. Then, for each

$$
\lambda \in] \frac{\Phi(\tilde{u})}{\Psi(\tilde{u})}, \frac{r}{\sup _{u \in \Phi^{-1}(]-\infty, r[)} \Psi(u)}[
$$


there is $u_{\lambda} \in \Phi^{-1}(] 0, r[)$ (hence $u_{\lambda} \neq 0$ ) such that $I_{\lambda}\left(u_{\lambda}\right)<I_{\lambda}(u)$ for all $u \in$ $\Phi^{-1}(] 0, r[)$ and $I_{\lambda}^{\prime}\left(u_{\lambda}\right)=0$.

Theorem 2.4 ([3], Theorem 3.2). Let $X$ be a real Banach space, and let $\Phi, \Psi: X \longrightarrow$ $\mathbb{R}$ be two continuously Gâteaux differentiable functionals such that $\Phi$ is bounded from below and

$\Phi(0)=\Psi(0)=0$. Fix $r>0$ such that $\sup _{u \in \Phi^{-1}(]-\infty, r[)} \Psi(u)<+\infty$ and assume that for each $\lambda \in] 0, \frac{r}{\sup _{u \in \Phi^{-1}(]-\infty, r[)} \Psi(u)}[$ the functional $\Phi-\lambda \Psi$ satisfies the $(P S)$ condition and it is unbounded from below. Then, for each

$$
\lambda \in] 0, \frac{r}{\sup _{u \in \Phi^{-1}(]-\infty, r[)} \Psi(u)}[
$$

the functional $I_{\lambda}$ admits two distinct critical points.

Now we present two propositions that will be needed to prove the main Theorems of this paper.

Proposition 2.5. Take $\Phi$ and $\Psi$ as in the definition 2.1 and fix $\lambda>0$. Then $I_{\lambda}=\Phi-\lambda \Psi$ satisfies the $(P S)^{[r]}$-condition for any $r>0$.

Proof. Consider sequence $\left\{u_{n}\right\} \subseteq W^{2,2}(\mathbb{R})$ such that $\left\{I_{\lambda}\left(u_{n}\right)\right\}$ is bounded,

$$
\lim _{n \rightarrow+\infty}\left\|I_{\lambda}^{\prime}\left(u_{n}\right)\right\|_{W^{2,2}(\mathbb{R})^{*}}=0
$$

and $\Phi\left(u_{n}\right)<r, \forall n \in \mathbb{N}$. Since $\Phi\left(u_{n}\right)<r$, we have, $\frac{1}{2}\left\|u_{n}\right\|^{2}<r$ and so $\left\{u_{n}\right\}$ is bounded in $W^{2,2}(\mathbb{R})$. Therefore passing to a subsequence if necessary we can assume that $u_{n}(x) \rightarrow u(x), x \in \mathbb{R}$ (from the compact embedding $W^{2,2}(\mathbb{R}) \rightarrow C([-T, T]), T>$ 0 ) and $\left\{u_{n}\right\}$ weakly converges to $u$ in $L^{\infty}(\mathbb{R})$ ( from the continuous embedding $\left.W^{2,2}(\mathbb{R}) \rightarrow L^{\infty}(\mathbb{R})\right)$ and hence there is $s>0$ such that $\left|u_{n}(x)\right| \leq s$ for a.e. $x \in \mathbb{R}$ and for all $n \in \mathbb{N}$. Now according to Lebesque's Dominated Convergence Theorem, Since $\alpha f\left(u_{n}(x)\right) \leq \alpha \cdot \max _{|\xi| \leq s} f(\xi) \in L^{1}(\mathbb{R})$ for all $n \in \mathbb{N}$ and $f\left(u_{n}(x)\right) \rightarrow f(u(x))$ for a.e. $x \in \mathbb{R}$ ( $f$ is continuous function), one has $\alpha f\left(u_{n}\right)$ is strongly converging to $\alpha f(u)$ in $L^{1}(\mathbb{R})$. Now since $u_{n} \rightarrow u$ in $L^{\infty}(\mathbb{R})$ and $\alpha f\left(u_{n}\right) \rightarrow \alpha f(u)$ in $L^{1}(\mathbb{R}) \subseteq\left(L^{\infty}(\mathbb{R})\right)^{*}$ then from [[4],proposition $3.5($ iv)], one has

$$
\lim _{n \rightarrow+\infty} \int_{\mathbb{R}} \alpha(x) f\left(u_{n}(x)\right)\left(u_{n}(x)-u(x)\right) d x=0 .
$$

From $\lim _{n \rightarrow+\infty}\left\|I_{\lambda}^{\prime}\left(u_{n}\right)\right\|_{W^{2,2}(\mathbb{R})^{*}}=0$, there exists a sequence $\left\{\varepsilon_{n}\right\}$, with $\varepsilon_{n} \rightarrow 0^{+}$, such that

$$
\left|\int_{\mathbb{R}}\left(u_{n}^{\prime \prime}(x) v^{\prime \prime}(x)-A u_{n}^{\prime}(x) v^{\prime}(x)+B u_{n}(x) v(x)-\lambda \alpha(x) f\left(u_{n}(x)\right) v(x)\right) d x\right| \leq \varepsilon_{n},
$$


for all $n \in \mathbb{N}$ and for all $v \in W^{2,2}(\mathbb{R})$ with $\|v\| \leq 1$. Taking into account $v(x)=$ $\frac{u_{n}(x)-u(x)}{\left\|u_{n}-u\right\|}$, from (9) one has

$$
\begin{gathered}
\mid \int_{\mathbb{R}}\left(u_{n}^{\prime \prime}(x)\left(u_{n}^{\prime \prime}(x)-u^{\prime \prime}(x)\right)-A u_{n}^{\prime}(x)\left(u_{n}^{\prime}(x)-u^{\prime}(x)\right)+B u_{n}(x)\left(u_{n}(x)-u(x)\right)-\right. \\
\left.\lambda \alpha(x) f\left(u_{n}(x)\right)\left(u_{n}(x)-u(x)\right)\right) d x \mid \leq \varepsilon_{n}\left\|u_{n}-u\right\|
\end{gathered}
$$

for all $n \in \mathbb{N}$.Now according to inequality $|a||b| \leq \frac{1}{2}|a|^{2}+\frac{1}{2}|b|^{2}$ we have

$$
\begin{gathered}
\int_{\mathbb{R}}\left(u_{n}^{\prime \prime}(x)\left(u_{n}^{\prime \prime}(x)-u^{\prime \prime}(x)\right)-A u_{n}^{\prime}(x)\left(u_{n}^{\prime}(x)-u^{\prime}(x)\right)+B u_{n}(x)\left(u_{n}(x)-u(x)\right)\right) d x \\
=\int_{\mathbb{R}}\left(\left|u_{n}^{\prime \prime}(x)\right|^{2}-A\left|u_{n}^{\prime}(x)\right|^{2}+B\left|u_{n}(x)\right|^{2}\right) d x-\int_{\mathbb{R}}\left(u_{n}^{\prime \prime}(x) u^{\prime \prime}(x)-A u_{n}^{\prime}(x) u^{\prime}(x)+B u_{n}(x) u(x)\right) \\
\geq\left\|u_{n}\right\|^{2}-\int_{\mathbb{R}}\left(\frac{1}{2}\left|u_{n}^{\prime \prime}(x)\right|^{2}+\frac{1}{2}\left|u^{\prime \prime}(x)\right|^{2}-\frac{1}{2} A\left|u_{n}^{\prime}(x)\right|^{2}\right. \\
\left.-\frac{1}{2} A\left|u^{\prime}(x)\right|^{2}+\frac{1}{2} B\left|u_{n}(x)\right|^{2}+\frac{1}{2} B|u(x)|^{2}\right) d x \\
=\left\|u_{n}\right\|^{2}-\frac{1}{2}\left\|u_{n}\right\|^{2}-\frac{1}{2}\|u\|^{2}=\frac{1}{2}\left\|u_{n}\right\|^{2}-\frac{1}{2}\|u\|^{2}
\end{gathered}
$$

Hence from (10), we have

$$
\frac{1}{2}\left\|u_{n}\right\|^{2}-\frac{1}{2}\|u\|^{2} \leq \lambda \int_{\mathbb{R}} \alpha(x) f\left(u_{n}(x)\right)\left(u_{n}(x)-u(x)\right) d x+\varepsilon_{n}\left\|u_{n}-u\right\|
$$

that is,

$$
\frac{1}{2}\left\|u_{n}\right\|^{2} \leq \frac{1}{2}\|u\|^{2}+\lambda \int_{\mathbb{R}} \alpha(x) f\left(u_{n}(x)\right)\left(u_{n}(x)-u(x)\right) d x+\varepsilon_{n}\left\|u_{n}-u\right\| .
$$

Taking into account (8),from (11) when $\varepsilon_{n} \rightarrow 0^{+}$, we have,

$$
\limsup _{n \rightarrow+\infty}\left\|u_{n}\right\| \leq\|u\|
$$

Thus [[4], proposition 3.32] ensures that $u_{n} \rightarrow u$, strongly in $W^{2,2}(\mathbb{R})$ and the proof is complete.

Proposition 2.6. Assume that there are $M>0$ and $\theta>2$ such that

$$
0<\theta F(\xi) \leq \xi f(\xi)
$$

for all $\xi \geq M$. Then $I_{\lambda}=\Phi-\lambda \Psi$ satisfies the $(P S)$-condition and it is unbounded from below. 
Proof. First we prove that $I_{\lambda}$ satisfies $(P S)$-condition for every $\lambda>0$. For this purpose we will prove that for arbitrary sequence $\left\{u_{n}\right\} \subset W^{2,2}(\mathbb{R})$ satisfying

$$
\begin{gathered}
\left|I_{\lambda}\left(u_{n}\right)\right| \leq D \text { for some } D>0 \text { and for all } n \in \mathbb{N}, \\
I_{\lambda}^{\prime}\left(u_{n}\right) \rightarrow 0 \text { in } W^{2,2}(\mathbb{R})^{*} \text { as } n \rightarrow \infty,
\end{gathered}
$$

contains a convergent subsequence. Let $\bar{u}_{n}(x)=\max \left\{0,-u_{n}(x)\right\}$. From (14) we have

$$
\left|I_{\lambda}^{\prime}\left(u_{n}\right)(v)\right| \leq \varepsilon_{n}\|v\|
$$

for all $v \in W^{2,2}(\mathbb{R})$ with $\varepsilon_{n} \rightarrow 0^{+}$. Hence $\left|I_{\lambda}^{\prime}\left(u_{n}\right)\left(\bar{u}_{n}\right)\right| \leq \varepsilon_{n}\left\|\bar{u}_{n}\right\|$, that is,

$$
\begin{array}{r}
\mid \int_{\mathbb{R}}\left(u_{n}^{\prime \prime}(x) \bar{u}_{n}^{\prime \prime}(x)-A u_{n}^{\prime}(x) \bar{u}_{n}^{\prime}(x)+B u_{n}(x) \bar{u}_{n}(x)-\lambda \int_{\mathbb{R}} \alpha(x) f\left(u_{n}(x)\right) \bar{u}_{n}(x) d x \mid\right. \\
\leq \varepsilon_{n}\left\|\bar{u}_{n}\right\| .
\end{array}
$$

Therefore

$$
\left\|\bar{u}_{n}\right\|^{2}+\lambda \int_{\mathbb{R}} \alpha(x) f\left(u_{n}(x)\right) \bar{u}_{n}(x) d x \leq \varepsilon_{n}\left\|\bar{u}_{n}\right\| .
$$

Hence $\left\|\bar{u}_{n}\right\| \leq \varepsilon_{n}$. Thus $\left\{\bar{u}_{n}\right\}$ strongly converges to 0 in $W^{2,2}(\mathbb{R})$ and so it is bounded in $W^{2,2}(\mathbb{R})$. Thus according to $(2)$ it is bounded in $L^{\infty}(\mathbb{R})$ and hence $0 \leq \bar{u}_{n}(x) \leq L$ for some $L \geq 0$ and for a.e. $x \in \mathbb{R}$ and one has,

$$
u_{n}(x) \geq-L
$$

for a.e. $x \in \mathbb{R}$ and for all $n \in \mathbb{N}$. Now we prove that $\left\{u_{n}\right\}$ is bounded in $W^{2,2}(\mathbb{R})$. For this end from (15), we have

$$
-I_{\lambda}^{\prime}\left(u_{n}\right)\left(u_{n}\right) \leq \varepsilon_{n}\left\|u_{n}\right\|
$$

for all $n \in \mathbb{N}$ with $\varepsilon_{n} \rightarrow 0^{+}$. Now we have

$$
\begin{aligned}
I_{\lambda}\left(u_{n}\right)-\frac{1}{\theta} I_{\lambda}^{\prime}\left(u_{n}\right)\left(u_{n}\right)=\left(\frac{1}{2}-\right. & \left.\frac{1}{\theta}\right)\left\|u_{n}\right\|^{2} \\
& +\frac{\lambda}{\theta} \int_{\mathbb{R}} \alpha(x)\left[f\left(u_{n}(x)\right) u_{n}(x)-\theta F\left(u_{n}(x)\right)\right] d x
\end{aligned}
$$

From (27), one has

$$
\int_{u_{n}(x) \geq M} \alpha(x)\left[f\left(u_{n}(x)\right) u_{n}(x)-\theta F\left(u_{n}(x)\right)\right] d x \geq 0 .
$$

On the other hand, in view of (16) we have 


$$
\begin{aligned}
\left|\int_{-L \leq u_{n}(x)<M} \alpha(x)\left[f\left(u_{n}(x)\right) u_{n}(x)-\theta F\left(u_{n}(x)\right)\right] d x\right| \\
\leq \int_{-L \leq u_{n}(x)<M} \alpha(x) \max _{\xi \in[-L, M]}[f(\xi) \xi-\theta F(\xi)] d x \\
\quad \leq \max _{\xi \in[-L, M]}[f(\xi) \xi-\theta F(\xi)] \cdot|\alpha|_{1} .
\end{aligned}
$$

Hence from (18)

$$
I_{\lambda}\left(u_{n}\right)-\frac{1}{\theta} I_{\lambda}^{\prime}\left(u_{n}\right)\left(u_{n}\right) \geq\left(\frac{1}{2}-\frac{1}{\theta}\right)\left\|u_{n}\right\|^{2}-\frac{\lambda}{\theta} \max _{\xi \in[-L, M]}[f(\xi) \xi-\theta F(\xi)] \cdot|\alpha|_{1} .
$$

Taking into account (13),(17), from (19), one has

$$
\left(\frac{1}{2}-\frac{1}{\theta}\right)\left\|u_{n}\right\|^{2}-\frac{\lambda}{\theta} \max _{\xi \in[-L, M]}[f(\xi) \xi-\theta F(\xi)] \cdot|\alpha|_{1} \leq D+\frac{\varepsilon_{n}}{\theta}\left\|u_{n}\right\|
$$

that is,

$$
\left(\frac{1}{2}-\frac{1}{\theta}\right)\left\|u_{n}\right\|^{2} \leq D+\frac{\varepsilon_{n}}{\theta}\left\|u_{n}\right\|+\frac{\lambda}{\theta} \max _{\xi \in[-L, M]}[f(\xi) \xi-\theta F(\xi)] \cdot|\alpha|_{1} .
$$

Thus, (20) ensures that $\left\{u_{n}\right\}$ is bounded in $W^{2,2}(\mathbb{R})$. Finally, as the argument used to prove Proposition 2.5, $\left\{u_{n}\right\}$ admits a convergent subsequence and so $I_{\lambda}$ satisfies $(P S)$-condition.

From (27), by standard computations, there are positive constants $c_{1}, c_{2}$ such that

$$
F(\xi) \geq c_{1} \xi^{\theta}-c_{2}
$$

for all $\xi \geq 0$. Fixed $u_{0} \in W^{2,2}(\mathbb{R})-\{0\}$, and suppose that $u_{0} \geq 0$. For each $t>1$, we have

$$
I_{\lambda}\left(t u_{0}\right)=\frac{1}{2}\left\|t u_{0}\right\|^{2}-\lambda \int_{\mathbb{R}} \alpha(x) \cdot F\left(t u_{0}\right) d x .
$$

Taking into account (21), one has

$$
I_{\lambda}\left(t u_{0}\right) \leq \frac{t^{2}}{2}\left\|u_{0}\right\|^{2}-\lambda c_{1} t^{\theta} \int_{\mathbb{R}} \alpha(x) \cdot u_{0}^{\theta} d x+\lambda c_{2} \int_{\mathbb{R}} \alpha(x) d x
$$

and since $\theta>2$, this condition guarantees that $I_{\lambda}$ is unbounded from below. 


\section{MAIN RESULTS}

Before presenting the main theorems of this section, we introduce notations that are related to some constants that will appear in the main results of this section. Put

$$
\begin{aligned}
k & =\left(\frac{2048}{27}-\frac{32}{9} A+\frac{13}{40} B\right)^{-1}, \\
\alpha_{0} & =\int_{\frac{3}{8}}^{\frac{5}{8}} \alpha(x) d x \\
E & =\frac{\int_{\frac{3}{8}}^{\frac{5}{8}} \alpha(x) d x}{\int_{\mathbb{R}} \alpha(x) d x}=\frac{\alpha_{0}}{|\alpha|_{1}}, \text { and hence } E \leq 1, \\
h & =C_{A, B}\left(\frac{2}{k}\right)^{\frac{1}{2}},
\end{aligned}
$$

and

$$
I=\frac{E}{h^{2}},
$$

where $C_{A, B}$ is given in proposition 2.1 .

Let us formulate the main results.

Theorem 3.1. Assume that there exist two positive constants $\eta$ and $\theta$ with $\eta<\theta$ such that

$$
\frac{F(\theta)}{\theta^{2}}<I \frac{F(\eta)}{\eta^{2}}
$$

Then, for each

$$
\lambda \in] \frac{1}{2|\alpha|_{1} C_{A, B}^{2}} \frac{1}{I} \frac{\eta^{2}}{F(\eta)}, \frac{1}{2|\alpha|_{1} C_{A, B}^{2}} \frac{\theta^{2}}{F(\theta)}[,
$$

problem $\left(P_{\lambda}\right)$ admits at least one non-trivial and non-negative weak solution $u_{0} \in$ $W^{2,2}(\mathbb{R})$ such that $\left|u_{0}\right|_{\infty}<\theta$.

Proof. Our aim is to apply Theorem 2.3, to problem $\left(P_{\lambda}\right)$. Fix $\lambda$, as in the conclusion. Take $X=W^{2,2}(\mathbb{R})$ and $\Phi$ and $\Psi$ as in the previous section. We observe that the regularity assumptions of Theorem 2.3 on $\Phi$ and $\Psi$ are satisfied and also according to proposition 2.5 , the functional $I_{\lambda}$ satisfies the $(P S)^{[r]}$-condition for all $r>0$.

Hence, our aim is to verify (7). Put

$$
r:=\frac{1}{2}\left(\frac{\theta}{C_{A, B}}\right)^{2}
$$


and

$$
w(x):= \begin{cases}-\frac{64 \eta}{9}\left(x^{2}-\frac{3}{4} x\right) & \text { if } x \in\left[0, \frac{3}{8}\right], \\ \eta & \text { if } \left.x \in] \frac{3}{8}, \frac{5}{8}\right], \\ -\frac{64 \eta}{9}\left(x^{2}-\frac{5}{4} x+\frac{1}{4}\right) & \text { if } \left.x \in] \frac{5}{8}, 1\right], \\ 0 & \text { otherwise. }\end{cases}
$$

We clearly observe that $w \in X$ and, in particular,

$$
\begin{gathered}
\Phi(w)=\frac{1}{2}\|w\|^{2}=\frac{1}{2} \int_{\mathbb{R}}\left(\left|w^{\prime \prime}(x)\right|^{2}-A\left|w^{\prime}(x)\right|^{2}+B|w(x)|^{2}\right) d x= \\
\eta^{2}\left(\frac{2048}{27}-\frac{32}{9} A+\frac{13}{40} B\right)=\frac{\eta^{2}}{k}=\frac{1}{2}\left(\frac{\eta h}{C_{A, B}}\right)^{2} .
\end{gathered}
$$

Moreover from $\frac{1}{I}=\frac{h^{2}}{E} \geq h^{2}, \eta<\theta$ and (22) we have,

$$
\eta h^{3} \frac{F(\theta)}{\theta^{2}} \leq \eta h \frac{1}{I} \frac{F(\theta)}{\theta^{2}}<\eta h \frac{1}{I} \cdot I \frac{F(\eta)}{\eta^{2}} \leq h \frac{F(\theta)}{\eta}
$$

that is, $\eta h<\theta$ and thus

$$
\Phi(w)=\frac{1}{2}\left(\frac{\eta h}{C_{A, B}}\right)^{2}<\frac{1}{2}\left(\frac{\theta}{C_{A, B}}\right)^{2}=r,
$$

Therefore, $0<\Phi(w)<r$. Now for each $u \in X$ and bearing (2) in mind, we see that

$$
\begin{array}{r}
\Phi^{-1}(]-\infty, r[)=\{u \in X ; \Phi(u)<r\}= \\
\left\{u \in X ; \frac{1}{2}\|u\|^{2}<\frac{1}{2}\left(\frac{\theta}{C_{A, B}}\right)^{2}\right\}= \\
\left\{u \in X ; C_{A, B}\|u\|<\theta\right\} \subseteq\left\{u \in X ;|u|_{\infty}<\theta\right\},
\end{array}
$$

and it follows that

$$
\begin{aligned}
\sup _{u \in \Phi^{-1}(]-\infty, r[)} \Psi(u) & =\sup _{u \in \Phi^{-1}(]-\infty, r[)} \int_{\mathbb{R}} \alpha(x) F(u(x)) d x \\
& \leq \int_{\mathbb{R}} \alpha(x) \sup _{|\xi|<\theta} F(\xi) d x=|\alpha|_{1} F(\theta) .
\end{aligned}
$$

Hence, we have

$$
\frac{\sup _{u \in \Phi^{-1}(]-\infty, r[)} \Psi(u)}{r} \leq \frac{|\alpha|_{1} F(\theta)}{\frac{1}{2}\left(\frac{\theta}{C_{A, B}}\right)^{2}}=2|\alpha|_{1} C_{A, B}^{2} \frac{F(\theta)}{\theta^{2}}<\frac{1}{\lambda} .
$$


On the other hand

$$
\begin{gathered}
\frac{\Psi(w)}{\Phi(w)}=\frac{\int_{\mathbb{R}} \alpha(x) F(w(x)) d x}{\frac{1}{2}\left(\frac{\eta h}{C_{A, B}}\right)^{2}} \geq \frac{\int_{\frac{3}{8}}^{\frac{5}{8}} \alpha(x) F(\eta) d x}{\frac{1}{2}\left(\frac{\eta h}{C_{A, B}}\right)^{2}}=\frac{\alpha_{0} F(\eta)}{\frac{1}{2}\left(\frac{\eta h}{C_{A, B}}\right)^{2}}= \\
2|\alpha|_{1} C_{A, B}^{2} \frac{E}{h^{2}} \frac{F(\eta)}{\eta^{2}}=2|\alpha|_{1} C_{A, B}^{2} I \frac{F(\eta)}{\eta^{2}}>\frac{1}{\lambda} .
\end{gathered}
$$

Now from (24) and (25) we have,

$$
\frac{\sup _{u \in \Phi^{-1}(]-\infty, r[)} \Psi(u)}{r}<\frac{\Psi(w)}{\Phi(w)}
$$

and (7) is proved. Finally, for each

$$
\lambda \in] \frac{1}{2|\alpha|_{1} C_{A, B}{ }^{2}} \frac{1}{I} \frac{\eta^{2}}{F(\eta)}, \frac{1}{2|\alpha|_{1} C_{A, B}{ }^{2}} \frac{\theta^{2}}{F(\theta)}[\subseteq] \frac{\Phi(w)}{\Psi(w)}, \frac{r}{\sup _{u \in \Phi^{-1}(]-\infty, r[)} \Psi(u)}[
$$

since the weak solutions of the problem $\left(P_{\lambda}\right)$ are exactly the solutions of the equation $I_{\lambda}^{\prime}(u)=0$, then Theorem 2.3 (with $\tilde{u}=w$ ) and lemma 2.2 will be guaranteed the conclusion.

Now, we present the following example to illustrate Theorem 3.1.

Example 3.1. Suppose that $f: \mathbb{R} \rightarrow \mathbb{R}$ is continuous and non-negative function and

$$
\alpha(x):= \begin{cases}4 & \text { if } x \in\left[\frac{3}{8}, \frac{5}{8}\right] \\ 0 & \text { otherwise. }\end{cases}
$$

Let $A=-1$ and $B=1$, then we have,

$k=\left(\frac{86111}{1080}\right)^{-1}, C_{A, B}=\frac{\sqrt{2}}{2}, h=\left(\frac{86111}{1080}\right)^{\frac{1}{2}}, E=1, I=\frac{1080}{86111}$. Also let $\eta=1$ and $\theta=10$. Now if

$$
\frac{1}{100} \int_{0}^{10} f(\xi) d \xi<\frac{1080}{86111} \int_{0}^{1} f(\xi) d \xi
$$

then according to Theorem 3.1 for each

$$
\lambda \in] \frac{86111}{1080 \int_{0}^{1} f(\xi) d \xi}, \frac{100}{\int_{0}^{10} f(\xi) d \xi}[
$$

problem

$$
\left\{\begin{array}{l}
u^{i v}(x)-u^{\prime \prime}(x)+u(x)=\lambda \alpha(x) f(u(x)) \quad, x \in \mathbb{R}, \\
u(-\infty)=u(+\infty)=0
\end{array}\right.
$$

has at least one non-trivial and non-negative weak solution $u_{0}$ such that $\left|u_{0}\right|_{\infty}<10$. 
Remark 3.1. For example in problem (26) we can consider,

$$
f(t):= \begin{cases}100 t^{2} & \text { if } t \leq 1 \\ -10000 t+10100 & \text { if } 1<t \leq 1.01 \\ 0 & \text { if } t>1.01\end{cases}
$$

It should be kept in mind that under certain suitable asymptotic conditions on the function $f$, existence a non-trivial weak solution will be guaranteed for problem $\left(P_{\lambda}\right)$ for any $\lambda \in \mathbb{R}^{+}$. In this case the following proposition is a consequence of Theorem 3.1 .

Corollary 3.2. Assume that

$$
\lim _{\xi \rightarrow 0^{+}} \frac{f(\xi)}{\xi}=+\infty \text { and } \lim _{\xi \rightarrow+\infty} \frac{f(\xi)}{\xi}=0 .
$$

Then, for each $\lambda \in \mathbb{R}^{+}$, problem $\left(P_{\lambda}\right)$ admits at least one non-trivial and nonnegative solution.

Proof. Suppose that $\lambda>0$ is fixed. Then from $\lim _{\xi \rightarrow 0^{+}} \frac{f(\xi)}{\xi}=+\infty$ we have

$\lim _{\xi \rightarrow 0^{+}} \frac{F(\xi)}{\xi^{2}}=+\infty$ and so $\frac{1}{\lambda}<2 \mid \alpha{ }_{1} C_{A, B}^{2} I \frac{F(\eta)}{\eta^{2}}$, for some $\eta>0$ such that sufficiently small.

On the other hand from $\lim _{\xi \rightarrow+\infty} \frac{f(\xi)}{\xi}=0$ we have

$\lim _{\xi \rightarrow+\infty} \frac{F(\xi)}{\xi^{2}}=0$ and so $2|\alpha|{ }_{1} C_{A, B}^{2} \frac{F(\theta)}{\theta^{2}}<\frac{1}{\lambda}$, for some $\theta>0$ with $\eta<\theta$ such that sufficiently large. Finally we can apply Theorem 3.1 and the conclusion follows.

Now, we point out the following existence results, as consequences of Theorem 2.4 .

Theorem 3.3. Suppose that there are $M>0$ and $\theta>2$ such that

$$
0<\theta F(\xi) \leq \xi f(\xi)
$$

for all $\xi \geq M$. Then for each $\lambda \in] 0, \lambda^{*}\left[\right.$ where $\lambda^{*}=\frac{1}{|\alpha|_{1} F\left(\sqrt{2} C_{A, B}\right)}$, problem $\left(P_{\lambda}\right)$ admits at least two distinct non-negative weak solutions.

Proof. Our aim is to apply Theorem 2.4, to problem $\left(P_{\lambda}\right)$. Put $r=1$ and fixed $\lambda \in] 0, \lambda^{*}[$. Let $X, \Phi$ and $\Psi$ be as given in the proof of Theorem 3.1. We observe that the regularity assumptions of Theorem 2.4 on $\Phi$ and $\Psi$ are satisfied and also according to proposition 2.6, the functional $I_{\lambda}$ satisfies the $(P S)$-condition and it is unbounded from below. Since for each $u \in X$ such that $u \in \Phi^{-1}(]-\infty, 1[)$ we have $\|u\|<\sqrt{2}$, hence from (2) one has 


$$
\begin{gathered}
\frac{\sup _{u \in \Phi^{-1}(]-\infty, r[)} \Psi(u)}{r}=\sup _{u \in \Phi^{-1}(]-\infty, 1[)} \Psi(u)=\sup _{u \in \Phi^{-1}(]-\infty, 1[)} \int_{\mathbb{R}} \alpha(x) F(u(x)) d x \leq \\
\int_{\mathbb{R}} \alpha(x) \sup _{|\xi|<\sqrt{2} C_{A, B}} F(\xi) d x=|\alpha|_{1} F\left(\sqrt{2} C_{A, B}\right)=\frac{1}{\lambda^{*}}<\frac{1}{\lambda} .
\end{gathered}
$$

From (28) we have

$$
\lambda \in] 0, \lambda^{*}[\subseteq] 0, \frac{r}{\sup _{u \in \Phi^{-1}(]-\infty, r[)} \Psi(u)}[.
$$

So all hypotheses of Theorem 2.4 are verified. Therefore, for each $\lambda \in] 0, \lambda^{*}[$, the functional $I_{\lambda}$ admits at least two distinct critical points which are, non-negative solutions of problem $\left(P_{\lambda}\right)$ and the proof is complete.

Example 3.2. Let $A=-1$ and $B=1$ and so $C_{A, B}=\frac{\sqrt{2}}{2}$. Also suppose that $\alpha(x)=e^{-x^{2}}$ and hence $|\alpha|_{1}=\int_{-\infty}^{+\infty} e^{-x^{2}} d x=\sqrt{\pi}$. Now if we consider $f(\xi)=1+\xi^{6}$, one has

$$
0<4\left(\xi+\frac{\xi^{7}}{7}\right) \leq \xi\left(1+\xi^{6}\right)
$$

for all $\xi \geq \sqrt[6]{7}$ and so $(27)$ is verified. Therefore since $f(0) \neq 0$ then according to Theorem 3.3 for each $\lambda \in] 0, \frac{7}{8 \sqrt{\pi}}[$, problem

$$
\left\{\begin{array}{l}
u^{i v}(x)-u^{\prime \prime}(x)+u(x)=\lambda e^{-x^{2}}\left(1+u(x)^{6}\right) \quad, x \in \mathbb{R} \\
u(-\infty)=u(+\infty)=0
\end{array}\right.
$$

admits at least two non-trivial and non-negative classical solutions.

\section{REFERENCES}

[1] A. Ambrosetti and P.H. Rabinowitz, Dual variational methods in critical point theory and applications, J. Funct. Analysis, 14 (1973), 349-381.

[2] G. Bonanno, A critical point theorem via the Ekeland variational principle, Nonlin. Analysis, 75 (2012), 2992-3007.

[3] G. Bonanno, Relations between the mountain pass theorem and local minima, Adv. Nonlin. Analysis, 1 (2012), 205-220.

[4] H. Brezis, Functional Analysis, Sobolev Spaces and Partial Differential Equations, Springer Science+Business Media, 2011, doi: 10.1007/978-0-387-70914-7,

[5] G. Bonanno, B. Di Bella, A boundary value problem for fourth-order elastic beam equations, J. Math. Anal. Appl., 343 (2008), 1166-1176. 
[6] G. Bonanno, B. Di Bella, Infinitely many solutions for a fourth-order elastic beam equations, Nonl. Differ. Equ. Appl., 18 (2011), 357-368.

[7] G. Bonanno, B. Di Bella, D. O'Regan, Non-trivial solutions for nonlinear fourthorder elastic beam equations, Comput. Math. Appl., 62 (2011), 1862-1869.

[8] V.I. Burenkov, Sobolev Spaces on Domains, Volume 137, Leipzig, Teubner, 1998.

[9] G. Han, Z. Xu, Multiple solutions of some nonlinear fourth-order beam equations, Nonlinear Anal., 68 (2008), 3646-3656.

[10] X.-L. Liu,W.-T. Li, Existence and multiplicity of solutions for fourth-order boundary values problems with parameters, J. Math. Anal. Appl., 327 (2007), 362-375.

[11] B. Ricceri, A general variational principle and some of its applications, J. Comput. Appl. Math., 113 (2000), 401-410.

[12] F. Wang, Y. An, Existence and multiplicity of solutions for a fourth-order elliptic equation, Boundary Value Problems, 2012 (2012). 
